\title{
Crystal structure of $(E)-N^{\prime}$-((1,8-dihydropyren-1- yl)-methylene)picolinohydrazide, $\mathrm{C}_{23} \mathrm{H}_{15} \mathrm{~N}_{3} \mathrm{O}$
}

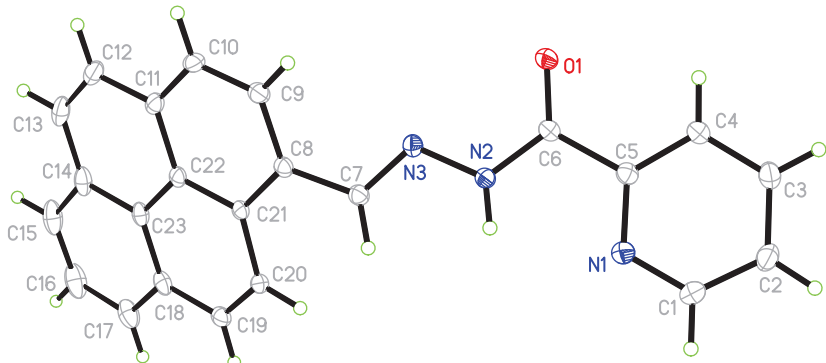

https://doi.org/10.1515/ncrs-2019-0297

Received April 24, 2019; accepted June 24, 2019; available online July 18, 2019

\section{Abstract}

$\mathrm{C}_{23} \mathrm{H}_{15} \mathrm{~N}_{3} \mathrm{O}$, monoclinic, $P 22_{1} / c$ (no. 14), $a=14.4372(3) \AA$, $b=11.9366(2) \AA, \quad c=10.23409(19) \AA, \quad \beta=106.963(2)^{\circ}$, $V=1686.92(6) \AA^{3}, Z=4, R_{\mathrm{gt}}(F)=0.0380, w R_{\mathrm{ref}}\left(F^{2}\right)=0.1055$, $T=115.2(3) \mathrm{K}$.

\section{CCDC no.: 1909229}

The asymmetric unit of the title structure is shown in the figure. Table 1 contains crystallographic data and Table 2 contains the list of the atoms including atomic coordinates and displacement parameters.

\section{Source of material}

All chemicals purchased were used without further purification. The title compound was synthesized from pyrene-1carbaldehyde by condensation reaction [5].

To a $100 \mathrm{~mL}$ flask, pyrene-1-carbaldehyde (1 mmol, $230 \mathrm{mg}$ ) was dispersed in $30 \mathrm{~mL}$ absolute ethanol. Under vigorously stirring, $20 \mathrm{~mL}$ colorless clarified solution containing picolinohydrazide ( $1 \mathrm{mmol}, 137 \mathrm{mg}$ ) was added to the above, two drops of acetic acid were also added as catalyst. After the addition, the stirred mixture was refluxed for $4 \mathrm{~h}$. At the beginning of stirring, a pale yellow clear solution formed. About one hour later, yellow precipitate appeared. The precipitate was filtered, washed 3 times with

\footnotetext{
*Corresponding author: Juan Yuan, Henan University of Chinese Medicine, Zhengzhou 450046, P.R. China, e-mail: hnzz_yuan@163.com

Yang Huanhuan, Xu Qingqing and Feng Haodong: Henan University of Chinese Medicine, Zhengzhou 450046, P.R. China
}

Table 1: Data collection and handling.

\begin{tabular}{ll}
\hline Crystal: & Yellow plate \\
Size: & $0.20 \times 0.10 \times 0.10 \mathrm{~mm}$ \\
Wavelength: & Cu $K \alpha$ radiation $(1.54184 \AA$ A $)$ \\
$\mu:$ & $0.69 \mathrm{~mm}^{-1}$ \\
Diffractometer, scan mode: & SuperNova, $\omega$ \\
$\theta_{\max }$, completeness: & $71.4^{\circ},>99 \%$ \\
$N(h k l)_{\text {measured }}, N(h k l)_{\text {unique }}, R_{\text {int }}:$ & $6586,3193,0.019$ \\
Criterion for $I_{\text {obs }}, N(h k l)_{\text {gt }}:$ & $I_{\text {obs }}>2 \sigma\left(I_{\text {obs }}\right), 2887$ \\
$N(\text { param })_{\text {refined }}:$ & 244 \\
Programs: & CrysAlix \\
& {$[3,4]$} \\
\hline
\end{tabular}

Table 2: Fractional atomic coordinates and isotropic or equivalent isotropic displacement parameters $\left(\AA^{2}\right)$.

\begin{tabular}{lrrrr}
\hline Atom & $\boldsymbol{x}$ & $\boldsymbol{y}$ & $\boldsymbol{z}$ & $\boldsymbol{U}_{\text {iso }} \boldsymbol{U}_{\text {eq }}$ \\
\hline O1 & $0.84562(6)$ & $0.28316(8)$ & $0.07460(8)$ & $0.0270(2)$ \\
N1 & $0.99204(8)$ & $0.16827(10)$ & $0.38576(11)$ & $0.0292(3)$ \\
N2 & $0.83471(7)$ & $0.29280(9)$ & $0.29246(10)$ & $0.0226(2)$ \\
H2 & 0.8614 & 0.2780 & 0.3774 & $0.027^{*}$ \\
N3 & $0.74812(7)$ & $0.35073(9)$ & $0.25153(10)$ & $0.0228(2)$ \\
C1 & $1.06772(10)$ & $0.10087(13)$ & $0.43467(14)$ & $0.0344(3)$ \\
H1 & 1.0874 & 0.0865 & 0.5280 & $0.041^{*}$ \\
C2 & $1.11881(10)$ & $0.05097(12)$ & $0.35510(15)$ & $0.0337(3)$ \\
H2A & 1.1711 & 0.0041 & 0.3938 & $0.040^{*}$ \\
C3 & $1.09007(11)$ & $0.07274(13)$ & $0.21693(15)$ & $0.0389(4)$ \\
H3 & 1.1225 & 0.0402 & 0.1602 & $0.047^{*}$ \\
C4 & $1.01222(10)$ & $0.14368(13)$ & $0.16336(14)$ & $0.0341(3)$ \\
H4 & 0.9923 & 0.1609 & 0.0707 & $0.041^{*}$ \\
C5 & $0.96500(8)$ & $0.18814(10)$ & $0.25122(12)$ & $0.0230(3)$ \\
C6 & $0.87669(8)$ & $0.25982(10)$ & $0.19658(12)$ & $0.0216(3)$ \\
C7 & $0.70558(9)$ & $0.36353(10)$ & $0.34455(12)$ & $0.0229(3)$ \\
H7 & 0.7343 & 0.3375 & 0.4328 & $0.028^{*}$ \\
C8 & $0.61136(8)$ & $0.41954(10)$ & $0.31002(11)$ & $0.0212(3)$ \\
C9 & $0.58717(10)$ & $0.49575(11)$ & $0.20178(12)$ & $0.0259(3)$ \\
H9 & 0.6337 & 0.5164 & 0.1599 & $0.031^{*}$ \\
C10 & $0.49550(10)$ & $0.54092(10)$ & $0.15602(12)$ & $0.0275(3)$ \\
H10 & 0.4817 & 0.5928 & 0.0851 & $0.033^{*}$ \\
C11 & $0.42331(9)$ & $0.51012(10)$ & $0.21417(12)$ & $0.0250(3)$ \\
C12 & $0.32611(10)$ & $0.55182(11)$ & $0.16378(14)$ & $0.0320(3)$ \\
H12 & 0.3107 & 0.6017 & 0.0908 & $0.038^{*}$ \\
C13 & $0.25685(10)$ & $0.52004(12)$ & $0.22011(15)$ & $0.0362(3)$ \\
H13 & 0.1946 & 0.5488 & 0.1854 & $0.043^{*}$ \\
C14 & $0.27669(9)$ & $0.44313(12)$ & $0.33208(15)$ & $0.0326(3)$ \\
C15 & $0.20523(11)$ & $0.40585(14)$ & $0.39038(19)$ & $0.0450(4)$ \\
& & & &
\end{tabular}


Table 2 (continued)

\begin{tabular}{lrrrr}
\hline Atom & $\boldsymbol{x}$ & $\boldsymbol{y}$ & $\boldsymbol{z}$ & $\boldsymbol{U}_{\text {iso }} \boldsymbol{U}_{\text {eq }}$ \\
\hline H15 & 0.1422 & 0.4324 & 0.3564 & $0.054^{*}$ \\
$\mathrm{C} 16$ & $0.22718(12)$ & $0.33066(14)$ & $0.4969(2)$ & $0.0485(4)$ \\
H16 & 0.1786 & 0.3063 & 0.5330 & $0.058^{*}$ \\
$\mathrm{C} 17$ & $0.32056(12)$ & $0.29072(13)$ & $0.55126(17)$ & $0.0401(4)$ \\
H17 & 0.3342 & 0.2406 & 0.6240 & $0.048^{*}$ \\
$\mathrm{C} 18$ & $0.39448(10)$ & $0.32521(11)$ & $0.49746(13)$ & $0.0279(3)$ \\
$\mathrm{C} 19$ & $0.49203(10)$ & $0.28662(10)$ & $0.55046(13)$ & $0.0277(3)$ \\
H19 & 0.5078 & 0.2395 & 0.6261 & $0.033^{*}$ \\
$\mathrm{C} 20$ & $0.56213(9)$ & $0.31664(10)$ & $0.49373(12)$ & $0.0232(3)$ \\
H20 & 0.6245 & 0.2888 & 0.5303 & $0.028^{*}$ \\
C21 & $0.54220(8)$ & $0.39069(10)$ & $0.37808(11)$ & $0.0197(2)$ \\
C22 & $0.44645(8)$ & $0.43457(10)$ & $0.32701(12)$ & $0.0212(3)$ \\
C23 & $0.37259(9)$ & $0.40128(10)$ & $0.38578(13)$ & $0.0248(3)$ \\
\hline
\end{tabular}

ethanol/water (1:1) to obtain yellow powder. Yield: $c a 85 \%$. The yellow powder $(0.05 \mathrm{mmol}, 17.5 \mathrm{mg})$ was dissolved in methanol/dichloromethane $(1: 1)(10 \mathrm{~mL})$ under stirring to give a pale yellow solution to evaporate slowly at room temperature. After three days, yellow plate crystals appeared. Yield: $c a 70 \%$.

\section{Experimental details}

The $\mathrm{H}$ atoms were added geometrically using riding models and refined isotropically. Their $U_{\text {iso }}$ values were set to $1.2 U_{\text {eq }}$ of the parent $\mathrm{C}$ and $\mathrm{N}$ atoms, and $1.5 U_{\text {eq }}$ of the parent $\mathrm{O}$ atoms.

\section{Comment}

Various transition-metal ions are important for the life of organisms [6]. Fluorescent probe and chemosensors have been widely focused to detect transition-metal ions, especially in living cells and in vivo [7-9]. To our knowledge, the fluorescent probes based on pyrene derivatives have not been widely reported $[5,10,11]$. Herein, we report a new pyrenederived compound that contains an amide functional group, a pyridine ring and a carbon-nitrogen double bond moiety that can coordinate to metal ions. The title compound may be the potential probe for certain metal ion.

The asymmetric unit of the title compound contains a neutral molecule (see the figure). The amide $\mathrm{C}=\mathrm{O}$ bond length is 1.2291(15) $\AA$. The bond length of C7-N3 is 1.2837(15) $\AA$, which showed that the form of a double-bond between $\mathrm{C} 7$ and N3 [12]. The dihedral angle between pyrene moiety and pyridine ring is $42.66^{\circ}$. There are obvious intermolecular hydrogen bonds between imine and carbonyl oxygen (D...A: $2.986 \AA ̊$ ), which link adjacent molecules into a one-dimensional supramolecular chain structure.

Acknowledgements: This work was supported by the project of scientific and technological in Henan Province (Project No. 172102310433).

\section{References}

1. Rigaku. CrysAlis PRO Software system, version 1. 171. 38. 43 f. Rigaku Oxford Diffraction (2015).

2. Dolomanov, O. V.; Bourhis, L. J.; Gildea, R. J.; Howard, J. A. K.; Puschmann, H.: OLEX2: a complete structure solution, refinement and analysis program. J. Appl. Crystallogr. 42 (2009) 339-341.

3. Sheldrick, G. M.: Crystal structure refinement with SHELXL. Acta Crystallogr. C71 (2015) 3-8.

4. Sheldrick, G. M.: SHELXT-integrated space-group and crystal-structure determination. Acta Crystallogr. A71 (2015) 3-8.

5. Zhou, Y.; Wang, F.; Kim, Y.; Kim, S.; Yoon, J.: $\mathrm{Cu}^{2+}$-selective ratiometric and "off-on" sensor based on the rhodamine derivative bearing pyrene group. Org. Lett. 11 (2009) 4442-4445.

6. Lippard, S. J.; Berg, J. M.: Principles of bioinorganic chemistry. University science books, Mill Valley, CA (1994).

7. Xi, P.; Dou, J.; Huang, L.; Xu, M.; Chen, F.; Wu, Y.; Bai, D.; Li, W.; Zeng, Z.: A selective turn-on fluorescent sensor for $\mathrm{Cu}(\mathrm{II})$ and its application in imaging in living cells. Sens. Actuators, B 148 (2010) 337-341.

8. Yang, H.; Zhou, Z.; Huang, K.; Yu, M.; Li, F.; Yi, T.; Huang, C.: Multisignaling optical-electrochemical sensor for $\mathrm{Hg}^{2+}$ based on a rhodamine derivative with a ferrocene unit. Org. Lett. 9 (2007) 4729-4732.

9. Diao, Q.; Ma, P.; Lv, L.; Li, T.; Sun, Y.; Wang, X.; Song, D.: A water-soluble and reversible fluorescent probe for $\mathrm{Al}^{3+}$ and $\mathrm{F}^{-}$ in living cells. Sens. Actuators B 229 (2016) 138-144.

10. Wang, F.; Nandhakumar, R.; Moon, J. H.; Kim, K. M.; Lee, J. Y.; Yoon, J.: Ratiometric fluorescent chemosensor for silver ion at physiological pH. Inorg. Chem. 50 (2011) 2240-2245.

11. Manandhar, E.; Broome, J. H.; Myrick, J.; Lagrone, W.; Cragg, P. J.; Wallace, K. J.: A pyrene-based fluorescent sensor for $\mathrm{Zn}^{2+}$ ions: a molecular 'butterfly'. Chem. Commun. 47 (2011) 8796-8798.

12. Saravanan, A.; Shyamsivappan, S.; Suresh, T.; Subashini, G.; Kadirvelu, K.; Bhuvanesh, N.; Nandhakumar, R.; Mohan, P. S.: An efficient new dual fluorescent pyrene based chemosensor for the detection of bismuth(III) and aluminium(III) ions and its applications in bio-imaging. Talanta 198 (2019) 249-256. 